\title{
Improved bound in Roth's theorem on arithmetic progressions
}

By

\author{
TOMASZ SchOEN*
}

\begin{abstract}
We prove that if $A \subseteq\{1, \ldots, N\}$ does not contain any non-trivial three-term arithmetic progression, then

$$
|A| \ll \frac{(\log \log N)^{3+o(1)}}{\log N} N .
$$
\end{abstract}

\section{Introduction}

In this paper we prove the following bound in Roth's theorem on arithmetic progressions.

Theorem 1 If $A \subseteq\{1, \ldots, N\}$ does not contain any non-trivial arithmetic progression of length three then

$$
|A| \ll \frac{(\log \log N)^{3}(\log \log \log N)^{5}}{\log N} N .
$$

The first non-trivial upper bound concerning the size of progression-free sets was given by Roth [18] who showed the above inequality with $N / \log \log N$. Then it was subsequently refined by Heath-Brown [14] and Szemerédi [26] with a denominator of $(\log N)^{c}$ for a positive constant $c$, by Bourgain [6, 7] and Sanders [21] by proving that bound with $c=1 / 2-o(1), c=2 / 3-o(1)$ and $c=3 / 4-o(1)$. Sanders [22] showed a result close to the logarithmic barrier

$$
|A| \ll \frac{(\log \log N)^{6}}{\log N} N
$$

and Bloom [4] further proved that

$$
|A| \ll \frac{(\log \log N)^{4}}{\log N} N
$$

for set $A \subseteq\{1, \ldots, N\}$ avoiding three-term arithmetic progressions. Recently a slightly weaker bound was obtained by a different argument by Bloom and Sisask [5]. Other results related to Roth's theorem can be found in [12], [15, [16], [24] and [25].

\footnotetext{
${ }^{*}$ The author is partially supported by National Science Centre, Poland grant 2019/35/B/ST1/00264
} 
Let us also comment on the recent progress for the analogous problem in a high-dimensional case. Croot, Lev and Pach [9] proved, by a polynomial method, an upper estimate $(4-c)^{n}$ with some constant $c>0$, for the size of progression-free sets in $(\mathbb{Z} / 4 \mathbb{Z})^{n}$. Later Ellenberg and Gijswijt [11] obtained the bound $(3-c)^{n}$ with a positive constant $c$ for subsets of $\mathbb{F}_{3}^{n}$. The latter result significantly improves the previous best bound of Bateman and Katz [1, however this the paper [1] contains many deep results and valuable ideas that could potentially be also used in the integer case.

Each of the mentioned papers contains significant novel ideas and methods, any of them are used in our proof of Theorem 1. We employ the density increment argument obtained via the Fourier analytical method invented by Roth [18]. We make use of the Bohr set machinery introduced by Bourgain [6]. We focus on the structure of the large spectrum, explored first by Bourgain [7] and thenceforth used in all further works. We also take advantage of deep insight into the structure of the large spectrum done by Bateman and Katz in [1] and [2].

Finally, let us mention that as far as the lower bound on the maximal size of progressionfree subsets of $\{1, \ldots, N\}$ is concerned, the first non-trivial lower estimate $N^{1-c(\log \log N)^{-1}}$ was established by Salem and Spencer [19]. Then Behrend [3] improved it to $\exp (-c \sqrt{\log N}) N$. Elkin [10] refined slightly Behrend's bound by a factor of $(\log N)^{1 / 2}$ and his argument was simplified in [13].

\section{Notation, Bohr sets and standard results}

All sets considered in the paper are finite subsets of $\mathbb{Z}$ or $\mathbb{Z} / N \mathbb{Z}$. We write $1_{A}(x)$ for the indicator function of set $A$. Given functions $f, g: \mathbb{Z} / N \mathbb{Z} \rightarrow \mathbb{C}$, the convolution of $f$ and $g$ is defined by

$$
(f * g)(x)=\sum_{t \in \mathbb{Z} / N \mathbb{Z}} f(t) g(x-t) .
$$

The Fourier coefficients of a function $f: \mathbb{Z} / N \mathbb{Z} \rightarrow \mathbb{C}$ are defined by

$$
\widehat{f}(r)=\sum_{x \in \mathbb{Z} / N \mathbb{Z}} f(x) e^{-2 \pi i x r / N}
$$

where $r \in \mathbb{Z} / N \mathbb{Z}$, and the above applies to the indicator function of $A \subseteq \mathbb{Z} / N \mathbb{Z}$ as well. Parseval's formula states in particular that

$$
\sum_{r=0}^{N-1}\left|\widehat{1_{A}}(r)\right|^{2}=|A| N .
$$

We also recall the fact that

$$
\left(\widehat{1_{A} * 1_{B}}\right)(r)=\widehat{1_{A}}(r) \widehat{1_{B}}(r) .
$$

For a real number $\theta \geqslant 0$, the $\theta$-spectrum of a set $A$ is the set

$$
\Delta_{\theta}(A)=\left\{r \in \mathbb{Z} / N \mathbb{Z}:\left|\widehat{1_{A}}(r)\right| \geqslant \theta|A|\right\} .
$$

For a specified set $A$ we often write $\Delta_{\theta}$ instead of $\Delta_{\theta}(A)$. 
For $m \in \mathbb{N}$ by $E_{2 m}(A)$ we denote the number of $2 m$-tuples $\left(a_{1}, \ldots, a_{m}, b_{1}, \ldots, b_{m}\right) \in A^{2 m}$ such that

$$
a_{1}+\cdots+a_{m}=b_{1}+\cdots+b_{m} .
$$

For $m=2$, we simply write $E(A)$ for $E_{4}(A)$ and we call it the additive energy of set $A$.

We define the span of a finite set $X$ by

$$
\operatorname{Span}(X)=\left\{\sum_{x \in X} \varepsilon_{x} x: \varepsilon_{x} \in\{-1,0,1\} \text { for all } x \in X\right\} .
$$

The $\operatorname{dimension} \operatorname{dim}(A)$ of set $A$ is the minimal size of set $X$ such that $A \subseteq \operatorname{Span}(X)$. The following theorem proven in [20] (see also [23] and [30]) provides an upper bound on the dimension of a set in terms of its additive doubling $K=|A+A| /|A|$.

Theorem 2 [20] Suppose that $|A+A|=K|A|$. Then $\operatorname{dim}(A) \ll K \log |A|$.

We are going to use a sophisticated concept of Bohr sets-a fundamental tool introduced to modern additive combinatorics by Bourgain [6].

Let $G=\mathbb{Z} / N \mathbb{Z}$ be a cyclic group and let us denote the group of its characters by $\widehat{G} \simeq \mathbb{Z} / N \mathbb{Z}$. We define the Bohr set with a generating set $\Gamma \subseteq \widehat{G}$ and a radius $\gamma \in\left(0, \frac{1}{2}\right]$ to be the set

$$
B(\Gamma, \gamma)=\{x \in \mathbb{Z} / N \mathbb{Z}:\|t x / N\| \leqslant \gamma \text { for all } t \in \Gamma\} .
$$

Here $\|\cdot\|$ denotes the distance to the integers, i.e. $\|x\|=\min _{y \in \mathbb{Z}}|x-y|$ for $x \in \mathbb{R}$. Given $\eta>0$ and a Bohr set $B=B(\Gamma, \gamma)$, by $B_{\eta}$ we mean the Bohr set $B(\Gamma, \eta \gamma)$. The two lemmas below are pretty standard, hence we refer the reader to [31] for a complete account. The size of $\Gamma$ is called the rank of $B$ and we denote it by $\operatorname{rk}(B)$.

Lemma 3 For every $\gamma \in\left(0, \frac{1}{2}\right]$ we have

$$
\gamma^{|\Gamma|} N \leqslant|B(\Gamma, \gamma)| \leqslant 8^{|\Gamma|+1}|B(\Gamma, \gamma / 2)|
$$

Bohr sets do not always behave like convex bodies. The size of Bohr sets can vary significantly even for small changes of the radius which was the motivation behind the following definition.

We call a Bohr set $B(\Gamma, \gamma)$ regular if for every $\eta$, with $|\eta| \leqslant 1 /(100|\Gamma|)$ we have

$$
(1-100|\Gamma||\eta|)|B| \leqslant\left|B_{1+\eta}\right| \leqslant(1+100|\Gamma||\eta|)|B| .
$$

Bourgain [6] showed that regular Bohr sets are ubiquitous.

Lemma 4 For every Bohr set $B(\Gamma, \gamma)$, there exists $\gamma^{\prime}$ such that $\frac{1}{2} \gamma \leqslant \gamma^{\prime} \leqslant \gamma$ and $B\left(\Gamma, \gamma^{\prime}\right)$ is regular.

The last lemma of this section presents a standard $L^{2}$ density increment technique introduced by Heath-Brown [14] and Szemerédi [26], see also [17]. A proof of the lemma below can be found in either of the following papers [21], 22] and [4]. 
Lemma 5 Let $A \subseteq \mathbb{Z} / N \mathbb{Z}$ be a set with density $\delta$. Let $\Gamma \subseteq \mathbb{Z} / N \mathbb{Z}$ and $\nu \geqslant 0$ be such that

$$
\sum_{r \in \Gamma \backslash\{0\}}\left|\widehat{1_{A}}(r)\right|^{2} \geqslant \nu|A|^{2}
$$

Then there is a regular Bohr set $B$ with $\operatorname{rk}(B)=\operatorname{dim}(\Gamma)$ and radius $\Omega\left((\operatorname{dim}(\Gamma))^{-1}\right)$ such that

$$
|(A+t) \cap B| \geqslant(1+\Omega(\nu)) \delta|B|
$$

for some $t$.

Throughout the paper we assume that set $A$ does not contain any non-trivial arithmetic progression of length three and that $N$ is a large number.

\section{Sketch of the argument}

We apply a widely used density increment argument introduced by Roth [18, however we use it in a rather non-standard way. In the first step, we increase the density by a large factor of the form $(\log (1 / \delta))^{1-o(1)}$ on some low-rank Bohr set. Then we apply the iterative method of Bloom to our new set with larger density to obtain the desired bound.

The general strategy can be roughly described as follows. Let $A \subseteq[N]$ be a set with density $\delta$ without arithmetic progressions of length three then it is known that $1_{A}$ must have large Fourier coefficients. To obtain a density increment we would have to find a small set $\Lambda$ such that $\operatorname{Span}(\Lambda)$ has large intersection with the spectrum $\Delta_{\delta}$. The size of $\Lambda$ is equal to the rank of a Bohr set, on which we will increase density, and density increment (given by the $L^{2}$ method) equals

$$
\left(1+\Omega\left(\delta^{2}\left|\operatorname{Span}(\Lambda) \cap \Delta_{\delta}\right|\right)\right) \delta .
$$

If we want to obtain the density increment by factor $\Omega(L)$ for some function $L \rightarrow \infty$, we have to locate $\Lambda$ of size $O\left(\delta^{-1+c}\right), c>0$ such that

$$
\left|\operatorname{Span}(\Lambda) \cap \Delta_{\delta}\right| \gg L \delta^{-2} .
$$

The main problem is that by of Bateman-Katz structural result (see Theorem 10) there are sets with spectrum such that the described set $\Lambda$ does not exist. Hence one needs to combine the above method with some new ideas.

In order to obtain the density increment we will consider three separate cases with respect to the size of Fourier coefficients of $1_{A}$ that in a sense dominate in $\Delta_{\delta^{1+\mu}}$ for a some small constant $\mu>0$. If the contribution of middle size or small Fourier coefficients is large we follow the method introduced by Bateman and Katz [1]. We consider essentially two subcases according to the additive behavior of the large spectrum $\Delta$. Following [1, we call the cases smoothing and nonsmoothing, respectively. If the higher energy $E_{8}(\Delta)$ is much bigger than one can deduce from the Hölder inequality applied to $E(\Delta)$ (smoothing case), then based on the Bateman-Katz argument we can indeed find a small set $\Lambda$ satisfying (11). The nonsmoothing case is more delicate. In that case we use a seminal result of Bateman and Katz [1, 2, that describes the structure of the spectrum in the nonsmoothing case and it turns out that again we can also find a small set 
$\Lambda$ satisfying (1), apart from one situation where roughly $\Delta \approx X+H,|\Delta| \sim \delta^{-3+O(\mu)},|X| \sim$ $\delta^{-2+O(\mu)},|H| \sim \delta^{-1+O(\mu)}$ and $H$ is a highly structured set. This case is considered separately in Lemma 13 which is an important part of our argument. We show that either the density can be increased on a Bohr set generated by $H$ (such a Bohr set has a very low rank) or $X$ contains additive substructure which again leads to a density increment on a low-rank Bohr set. The above argument does not apply when $\Delta_{\delta^{1+\mu}}$ is dominated by large Fourier coefficients. Then assuming that there are very few smaller Fourier coefficients in $\Delta_{\delta^{1+\mu}}$, using a different technique based on Fourier approximation method, we prove that $A$ does indeed have density increment on a low-rank Bohr set.

\section{Middle size Fourier coefficients}

Assume that $A \subseteq\left\{1, \ldots, N^{\prime}\right\}$ does not contain any non-trivial arithmetic progressions of length three. Let $N$ be any prime number satisfying $2 N^{\prime}<N \leqslant 4 N^{\prime}$. We embed $A$ in $\mathbb{Z} / N \mathbb{Z}$ in a natural way and observe that $A$ also does not contain any non-trivial arithmetic progression of length 3 in $\mathbb{Z} / N \mathbb{Z}$. Let us recall a standard argument that shows that $1_{A}$ must have large Fourier coefficients. The number of three-term arithmetic progressions in $A$ (including trivial ones) is expressed by the sum $\frac{1}{N} \sum_{r=0}^{N-1} \widehat{1_{A}}(r)^{2} \widehat{1_{A}}(-2 r)$, whence we have

$$
\frac{1}{N} \sum_{r=0}^{N-1} \widehat{1_{A}}(r)^{2} \widehat{1_{A}}(-2 r)=|A| .
$$

Clearly, we can assume that $|A|>\sqrt{2 N}$, so by the Hölder inequality

$$
\sum_{r \neq 0}\left|\widehat{1_{A}}(r)\right|^{3} \geqslant|A|^{3}-N|A| \geqslant \frac{1}{2}|A|^{3} .
$$

Since

$$
\sum_{r \notin \Delta_{\delta / 4}(A)}\left|\widehat{1_{A}}(r)\right|^{3} \leqslant \frac{1}{4} \delta|A| \sum_{r=0}^{N-1}\left|\widehat{1_{A}}(r)\right|^{2}=\frac{1}{4}|A|^{3}
$$

it follows that

$$
\sum_{r \in \Delta_{\delta / 4} \backslash\{0\}}\left|\widehat{1_{A}}(r)\right|^{3} \geqslant \frac{1}{4}|A|^{3},
$$

hence there are non-trivial Fourier coefficients with $\left|\widehat{1_{A}}(r)\right| \gg \delta|A|$.

However to obtain a large density increment we have to control Fourier coefficients below typical treshold $\delta|A|$. We will consider three separate cases:

$$
\begin{array}{r}
\sum_{r: \delta^{1-\mu}|A| \leqslant\left|\widehat{1_{A}}(r)\right| \leqslant \delta^{1 / 10}|A|}\left|\widehat{1_{A}}(r)\right|^{3} \geqslant \frac{1}{10} \delta^{\mu / 5}|A|^{3}, \\
\sum_{r: \delta^{1+\mu}|A| \leqslant\left|\widehat{1_{A}}(r)\right| \leqslant \delta^{1-\mu}|A|}\left|\widehat{1_{A}}(r)\right|^{3} \geqslant \frac{1}{10} \delta^{\mu / 5}|A|^{3},
\end{array}
$$


and the last one if (2) and (3) do not hold, where $\mu$ is a small positive constant. Throughout the paper we assume that $\delta^{\mu / 20}<\log ^{-1}(1 / \delta)$ since we know that $\delta \rightarrow 0$ as $N \rightarrow \infty$.

By dyadic argument, we obtain

$$
\sum_{r: \theta|A| \leqslant\left|\widehat{1_{A}}(r)\right| \leqslant 2 \theta|A|}\left|\widehat{1_{A}}(r)\right|^{3} \geqslant \frac{1}{10} \delta^{\mu / 5}|A|^{3} \log ^{-1}(1 / \delta)
$$

for some $\delta^{1-\mu} \leqslant \theta \leqslant \delta^{1 / 10}$, so

$$
\left|\Delta_{\theta}\right| \gg \theta^{-3} \delta^{\mu / 5} \log ^{-1}(1 / \delta) \geqslant \theta^{-3} \delta^{\mu / 4}
$$

In this section we consider the first case (2). We will apply the Bateman-Katz-Bloom lemma, see Lemma 5.3 in [1] and Theorem 4.1 in [4] (a slightly weaker version of Lemma 6 can be easily deduced from Lemma 8 ).

Lemma 6 Let $A \subseteq \mathbb{Z} / N \mathbb{Z}$ be a set with density $\delta$, and let $\Delta$ be a subset of $\Delta_{\theta}$. Then there exists a set $\Delta^{\prime} \subseteq \Delta$ such that $\left|\Delta^{\prime}\right| \gg \theta|\Delta|$ and $\operatorname{dim}\left(\Delta^{\prime}\right) \ll \theta^{-1} \log (1 / \delta)$.

Lemma 7 Let $A \subseteq \mathbb{Z} / N \mathbb{Z}$ be a set with density $\delta$, and suppose that (5) holds for some $\delta^{1-\mu} \leqslant$ $\theta \leqslant \delta^{1 / 10}$. Then there is a regular Bohr set $B$ with $\operatorname{rk}(B) \ll \delta^{-1+\mu / 3}$ and radius $\Omega\left(\delta^{1-\mu / 3}\right)$ such that for some $t$

$$
|(A+t) \cap B| \gg \delta^{1-\mu / 4}|B|
$$

Proof. By Lemma 6 there exists a set $\Delta_{1} \subseteq \Delta_{\theta}$ such that $\left|\Delta_{1}\right|=\Theta\left(\theta\left|\Delta_{\theta}\right|\right)$ and

$$
\operatorname{dim}\left(\Delta_{1}\right) \ll \theta^{-1} \log (1 / \delta) .
$$

By iterative application of Lemma [6, we see that there are disjoint sets $\Delta_{1}, \ldots, \Delta_{k} \subseteq \Delta_{\theta}$, for $k=\Theta\left(\delta^{-\mu / 2}\right)$ such that $\left|\Delta_{i}\right|=\Theta\left(\theta\left|\Delta_{\theta}\right|\right)$ and

$$
\operatorname{dim}\left(\Delta_{i}\right) \ll \theta^{-1} \log (1 / \delta)
$$

for every $1 \leqslant i \leqslant k$. Put $\Gamma=\bigcup_{i=1}^{k} \Delta_{i} \subseteq \Delta_{\theta}$ then by (15) we have

$$
|\Gamma| \gg \delta^{-\mu / 2} \theta^{-2} \delta^{\mu / 4} \gg \delta^{-\mu / 4} \theta^{-2}
$$

and

$$
\operatorname{dim}(\Gamma) \ll \delta^{-\mu / 2} \theta^{-1} \log (1 / \delta) \ll \delta^{-1+\mu / 2} \log (1 / \delta) \ll \delta^{-1+\mu / 3} .
$$

Therefore, by Lemma 5 a shift of the set $A$ has density at least

$$
\left(1+\Omega\left(\theta^{2}|\Gamma|\right)\right) \delta \gg \delta^{-1+\mu / 3}
$$

on a regular Bohr set with rank $O\left(\delta^{-1+\mu / 3}\right)$ and radius $\Omega\left(\delta^{1-\mu / 3}\right)$. 


\section{$5 \quad$ Additively smoothing spectrum}

In sections 5 and 6 we obtain a density increment provided that (3) holds. Hence for some $\delta^{1+\mu} \leqslant \theta \leqslant \delta^{1-\mu}$ we have

$$
\sum_{r: \theta|A| \leqslant\left|\widehat{1_{A}}(r)\right| \leqslant 2 \theta|A|}\left|\widehat{1_{A}}(r)\right|^{3} \gg|A|^{3} \log ^{-1}(1 / \delta) .
$$

Thus

$$
\left|\Delta_{\theta}\right| \geqslant \delta^{\mu / 5} \theta^{-3} \log ^{-1}(1 / \delta) \geqslant \delta^{2 \mu} \theta^{-2} \delta^{-1},
$$

so the size of $\Delta_{\theta}$ is close to the maximal possible value.

A well-known theorem of Shkredov [28, 29] states that for every $\Delta \subseteq \Delta_{\theta}$ and $m \in \mathbb{N}$ we have

$$
E_{2 m}(\Delta) \geqslant \theta^{2 m} \delta|\Delta|^{2 m} \text {. }
$$

Observe that by the Parseval formula $\left|\Delta_{\theta}\right| \leqslant \theta^{-2} \delta^{-1}$, so if we additionally assume that $\left|\Delta_{\theta}\right| \gg$ $\theta^{-2} \delta^{-1}$, then the Hölder inequality implies that for $m \geqslant 3$

$$
E_{2 m}\left(\Delta_{\theta}\right) \geqslant E\left(\Delta_{\theta}\right)^{m-1}\left|\Delta_{\theta}\right|^{m-2} \gg_{m} \theta^{2 m} \delta\left|\Delta_{\theta}\right|^{2 m},
$$

which essentially meets Shkredov's bound. This observation motivates the next definition introduced by Bateman and Katz. We say that a spectrum $\Delta_{\theta}$ is $\sigma$-additively smoothing (or simply additively smoothing if $\sigma$ is indicated) if

$$
E_{8}\left(\Delta_{\theta}\right) \geqslant \delta^{-\sigma} \theta^{8} \delta\left|\Delta_{\theta}\right|^{8}
$$

Otherwise, we say that the spectrum $\Delta_{\theta}$ is $\sigma$-additively nonsmoothing. In this section, we will obtain a density increment for additively smoothing spectrum.

The following lemma, proven in [23] (see Corollary 7.5) is an abelian group version of Bateman-Katz Lemma 5.3. The proof of this result requires some modifications, but similarly as in Bloom's Theorem 4.1 in [4] it relies on a probabilistic argument of Bateman and Katz.

Lemma 8 Let $\Delta \subseteq \mathbb{Z} / N \mathbb{Z}$ be a set such that $E_{2 s}(\Delta)=\kappa|\Delta|^{2 s} \geqslant 10^{s} s^{2 s}|\Delta|^{s}$, where $2 \leqslant s=$ $\lfloor\log |\Delta|\rfloor$. Then there exists a set $\Lambda \subseteq \Delta$ such that $|\Lambda| \ll \kappa^{-1 / 2 s} \log ^{3 / 2}|\Delta|$ and

$$
|\operatorname{Span}(\Lambda) \cap \Delta| \gg \kappa^{1 / 2 s}|\Delta| \log ^{-3 / 2}|\Delta|
$$

Lemma 9 Let $A \subseteq \mathbb{Z} / N \mathbb{Z},|A|=\delta N$ and suppose that for some $\delta^{1+\mu} \leqslant \theta \leqslant \delta^{1-\mu}$ we have $E_{8}\left(\Delta_{\theta}\right) \geqslant \delta^{-20 \mu} \theta^{8} \delta\left|\Delta_{\theta}\right|^{8}$. Then there is a regular Bohr set $B$ with rank $\operatorname{rk}(B) \ll \delta^{-1+\mu / 2}$ and radius $\Omega\left(\delta^{1-\mu / 2}\right)$ such that for some $t$

$$
|(A+t) \cap B| \gg \delta^{1-\mu / 2}|B|
$$


Proof. Put $s=\left\lfloor\log \left|\Delta_{\theta}\right|\right\rfloor$. Using the Hölder inequality and (5) we have

$$
\begin{aligned}
E_{2 s}\left(\Delta_{\theta}\right) & \geqslant E_{8}\left(\Delta_{\theta}\right)^{\frac{s-1}{3}}\left|\Delta_{\theta}\right|^{-\frac{s-4}{3}} \geqslant \delta^{O(1)} \delta^{\frac{1}{3}(1-20 \mu) s} \theta^{\frac{8}{3} s}\left|\Delta_{\theta}\right|^{\frac{7}{3} s} \\
& \geqslant \delta^{-\frac{1}{3}(18 \mu+o(1)) s} \theta^{2 s}\left|\Delta_{\theta}\right|^{2 s} \geqslant \delta^{-4 \mu s} \theta^{2 s}\left|\Delta_{\theta}\right|^{2 s}
\end{aligned}
$$

provided that $N$ is large enough. Notice that ([6) implies that $E_{2 s}\left(\Delta_{\theta}\right) \gg 10^{s} s^{2 s}\left|\Delta_{\theta}\right|^{s}$, so we can apply Lemma 8 . Thus, there exists a set $\Lambda$ such that

$$
|\Lambda| \ll \delta^{2 \mu} \theta^{-1} \log ^{3 / 2}(1 / \delta) \ll \delta^{-1+\mu / 2}
$$

and

$$
\left|\operatorname{Span}(\Lambda) \cap \Delta_{\theta}\right| \gg \delta^{-2 \mu} \theta \log ^{-3 / 2}(1 / \delta)\left|\Delta_{\theta}\right| \gg \delta^{-2 \mu} \theta^{-2} \log ^{-5 / 2}(1 / \delta) \gg \delta^{-\mu / 2} \theta^{-2}
$$

Now it is enough to use Lemma 5 with $\Gamma=\operatorname{Span}(\Lambda)$ to get the required result.

\section{Additively nonsmoothing spectrum}

In this section, we will obtain a density increment in a more difficult case, when the spectrum $\Delta_{\theta}$ is an additively nonsmoothing set. Recall that for some $\delta^{1+\mu} \leqslant \theta \leqslant \delta^{1-\mu}$ we have

$$
\left|\Delta_{\theta}\right| \geqslant \delta^{2 \mu} \theta^{-2} \delta^{-1}
$$

Bateman and Katz [1, 2] proved the following fundamental result characterizing the structure of additively nonsmoothing sets.

Theorem 10 Let $\tau>0$ be a fixed number. There exists a function $f=f_{\tau}:(0,1) \rightarrow(0, \infty)$ with $f(x) \rightarrow 0$ as $x \rightarrow 0$ such that the following holds. Let $\Delta$ be a symmetric set of an abelian group and let $\sigma>0$. Assume that $E\left(\Delta^{\prime}\right) \gg|\Delta|^{2+\tau}$ for every $\Delta^{\prime} \subseteq \Delta$ with $\left|\Delta^{\prime}\right| \gg|\Delta|$ and that $E_{8}(\Delta) \leqslant|\Delta|^{4+3 \tau+\sigma}$. Then there exists $\alpha, 0 \leqslant \alpha \leqslant \frac{1-\tau}{2}$, such that for $i=1, \ldots,\left\lceil|\Delta|^{\alpha-f(\sigma)}\right\rceil$ there are sets $H_{i}, X_{i}$ and $\Delta_{i} \subseteq \Delta$ such that

$$
\begin{aligned}
\left|H_{i}\right| & \ll|\Delta|^{\tau+\alpha+f(\sigma)}, \\
\left|X_{i}\right| & \ll|\Delta|^{1-\tau-2 \alpha+f(\sigma)}, \\
\left|H_{i}+H_{i}\right| & \ll\left|H_{i}\right|^{1+f(\sigma)},
\end{aligned}
$$

and

$$
\left|\left(X_{i}+H_{i}\right) \cap \Delta_{i}\right| \gg|\Delta|^{1-\alpha-f(\sigma)} .
$$

Furthermore, the sets $\Delta_{i}$ are pairwise disjoint.

We will apply Theorem 10 to the set $\Delta_{\theta}$. By Shkredov's theorem for every $\Delta \subseteq \Delta_{\theta}$ with $|\Delta| \gg\left|\Delta_{\theta}\right|$ we have

$$
E(\Delta) \geqslant \theta^{4} \delta|\Delta|^{4} \gg \delta^{10 \mu / 3} \theta^{2 / 3} \delta^{-2 / 3}|\Delta|^{7 / 3} \geqslant \delta^{4 \mu}|\Delta|^{7 / 3} \gg\left|\Delta_{\theta}\right|^{7 / 3-2 \mu} .
$$


On the other hand, by Lemma 9 we can assume that

$$
E_{8}\left(\Delta_{\theta}\right) \leqslant \delta^{-20 \mu} \theta^{8} \delta\left|\Delta_{\theta}\right|^{8} \leqslant\left|\Delta_{\theta}\right|^{5+10 \mu} .
$$

Therefore we can apply Theorem 10 with

$$
\tau=1 / 3-2 \mu \quad \text { and } \quad \sigma=16 \mu
$$

hence our spectrum $\Delta_{\theta}$ has structure described in Theorem 10 .

Throughout the paper assume that $f(\sigma) \geqslant \sigma$ and that $\mu$ and $f=f(16 \mu)$ are small constants.

Each of the four inequalities given in Theorem 10 is crucial in our approach. Note that from (17), (8) and (10) we can deduce lower bounds for the size of $H_{i}$ and $X_{i}$. In order to apply the last one, we will need the following simple, elementary lemma.

Lemma 11 Let $c, \varepsilon>0$ be such that $|(X+H) \cap \Delta| \geqslant c|X||H|^{1-\varepsilon}$. Then there is a set $X^{\prime} \subseteq X$ such that $\left|X^{\prime}\right| \geqslant \frac{c}{4}|X||H|^{-\varepsilon}$ and for every $Y \subseteq X^{\prime}$ we have $|(Y+H) \cap \Delta| \geqslant \frac{c^{2}}{8}|Y||H|^{1-2 \varepsilon}$.

Proof. Put $S=(X+H) \cap \Delta$ and notice that

$$
\sum_{t \in X+H}\left(1_{X} * 1_{H}\right)(t)=|X||H|
$$

Let us denote by $P$ the set of elements $t$ with $\left(1_{X} * 1_{H}\right)(t) \geqslant \frac{2}{c}|H|^{\varepsilon}$. Clearly, $|P| \leqslant \frac{c}{2}|X||H|^{1-\varepsilon}$ and therefore

$$
\sum_{t \in S \backslash P}\left(1_{X} * 1_{H}\right)(t)=\sum_{x \in X}|(x+H) \cap(S \backslash P)| \geqslant|S|-|P| \geqslant \frac{c}{2}|X||H|^{1-\varepsilon} .
$$

Let $X^{\prime}$ be the set of all $x \in X$ satisfying the inequality $|(x+H) \cap(S \backslash P)| \geqslant \frac{c}{4}|H|^{1-\varepsilon}$. Observe that

$$
\sum_{x \in X \backslash X^{\prime}}|(x+H) \cap(S \backslash P)| \leqslant \frac{c}{4}|H|^{1-\varepsilon}\left|X \backslash X^{\prime}\right| \leqslant \frac{c}{4}|X||H|^{1-\varepsilon},
$$

hence

$$
\left|X^{\prime}\right||H| \geqslant \sum_{x \in X^{\prime}}|(x+H) \cap(S \backslash P)| \geqslant \frac{c}{4}|X||H|^{1-\varepsilon} .
$$

Thus, $\left|X^{\prime}\right| \geqslant \frac{c}{4}|X||H|^{-\varepsilon}$ and if $Y \subseteq X^{\prime}$, then

$$
|(Y+H) \cap \Delta| \geqslant \frac{\frac{c}{4}|Y||H|^{1-\varepsilon}}{\frac{2}{c}|H|^{\varepsilon}},
$$

which yields to the required inequality.

The next lemma provides a density increment in a simpler case-in Bateman-Katz theorem we have $\alpha \geqslant 20 f$. 
Lemma 12 Let $A \subseteq \mathbb{Z} / N \mathbb{Z},|A|=\delta N$, and assume that for every $\Delta^{\prime} \subseteq \Delta$ with $\left|\Delta^{\prime}\right| \gg|\Delta|$ we have $E\left(\Delta^{\prime}\right) \gg|\Delta|^{7 / 3-2 \mu}$ and $E_{8}(\Delta) \leqslant|\Delta|^{5+10 \mu}$. Then either there is a regular Bohr set $B$ with $\operatorname{rk}(B) \ll \delta^{-1+f}$ and radius $\Omega\left(\delta^{1-f}\right)$ such that

$$
|(A+t) \cap B| \gg \delta^{1-f}|B|
$$

for some $t$; or there are sets $H$ and $X$ such that $|H| \ll\left|\Delta_{\theta}\right|^{1 / 3+21 f},|H+H| \ll|H|^{1+f},|X| \ll$ $\left|\Delta_{\theta}\right|^{2 / 3+2 f}$, and

$$
\left|(X+H) \cap \Delta_{\theta}\right| \gg\left|\Delta_{\theta}\right|^{1-21 f} .
$$

Proof. By Theorem 10 applied with $\tau=1 / 3-2 \mu$ there exist $0 \leqslant \alpha \leqslant 1 / 3+\mu$ and sets $H_{i}, X_{i}$ for $1 \leqslant i \leqslant\left\lceil\left|\Delta_{\theta}\right|^{\alpha-f}\right\rceil$ such that

$$
\left|\Delta_{\theta}\right|^{1 / 3-2 \mu+\alpha-2 f} \ll\left|H_{i}\right| \ll\left|\Delta_{\theta}\right|^{1 / 3-2 \mu+\alpha+f}
$$

and

$$
\left|\Delta_{\theta}\right|^{2 / 3+2 \mu-2 \alpha-2 f} \ll\left|X_{i}\right| \ll\left|\Delta_{\theta}\right|^{2 / 3+2 \mu-2 \alpha+f},
$$

that fulfill inequalities (7)-(10). First, we assume that $1 / 3-20 f \leqslant \alpha \leqslant 1 / 3+\mu$ and put $k=\left\lceil\left|\Delta_{\theta}\right|^{\alpha-25 f}\right\rceil$. Then by (10), (9) and Theorem 2 we have

$$
\left|\bigcup_{i=1}^{k}\left(X_{i}+H_{i}\right) \cap \Delta_{\theta}\right| \gg\left|\Delta_{\theta}\right|^{\alpha-25 f}\left|\Delta_{\theta}\right|^{1-\alpha-f} \geqslant\left|\Delta_{\theta}\right|^{1-f} \geqslant \theta^{-2} \delta^{-f}
$$

and

$$
\begin{aligned}
\operatorname{dim}\left(\bigcup_{i=1}^{k}\left(X_{i}+H_{i}\right)\right) & \ll \sum_{i=1}^{k}\left|X_{i}\right| \operatorname{dim}\left(H_{i}\right) \leqslant\left|\Delta_{\theta}\right|^{\alpha-25 f}\left|X_{i}\right|\left|H_{i}\right|^{f} \log \left|H_{i}\right| \\
& \ll\left|\Delta_{\theta}\right|^{2 / 3+2 \mu-\alpha-22 f} \leqslant\left|\Delta_{\theta}\right|^{1 / 3-f} \ll \delta^{-1+f} .
\end{aligned}
$$

Next let us assume that $20 f \leqslant \alpha \leqslant 1 / 3-20 f$. Observe that by (10) for every $i$ we have

$$
\left|\left(X_{i}+H_{i}\right) \cap \Delta_{i}\right| \gg\left|\Delta_{\theta}\right|^{1-\alpha-f} \gg\left|X_{i}\right|\left|H_{i}\right|\left|\Delta_{\theta}\right|^{-3 f} \gg\left|X_{i}\right|\left|H_{i}\right|^{1-5 f} .
$$

By Lemma 11 applied with $X_{i}, H_{i}$ and $\varepsilon=5 f$ there is $X_{i}^{\prime} \subseteq X_{i}$ such that

$$
\left|X_{i}^{\prime}\right| \gg\left|X_{i}\right|\left|H_{i}\right|^{-5 f} \gg\left|\Delta_{\theta}\right|^{2 / 3+2 \mu-2 \alpha-5 f} \geqslant\left|\Delta_{\theta}\right|^{1 / 3-\alpha+15 f} .
$$

Let $Y_{i} \subseteq X_{i}^{\prime}$ be any subset of size $\left\lceil\left|\Delta_{\theta}\right|^{1 / 3-\alpha+15 f}\right\rceil$. By Lemma [11, we have

$$
\left|\left(Y_{i}+H_{i}\right) \cap \Delta_{i}\right| \gg\left|\Delta_{\theta}\right|^{2 / 3-2 \mu+3 f} \geqslant\left|\Delta_{\theta}\right|^{2 / 3+f} \geqslant \theta^{-2} \delta^{-f} .
$$

Again Theorem 2 and (9) imply that

$$
\begin{aligned}
\operatorname{dim}\left(Y_{i}+H_{i}\right) & \leqslant \operatorname{dim}\left(Y_{i}\right) \operatorname{dim}\left(H_{i}\right) \ll\left|\Delta_{\theta}\right|^{1 / 3-\alpha+15 f}\left|H_{i}\right|^{f} \log \left|H_{i}\right| \\
& \leqslant\left|\Delta_{\theta}\right|^{1 / 3-\alpha+17 f} \leqslant\left|\Delta_{\theta}\right|^{1 / 3-f} \leqslant \delta^{-1+f} .
\end{aligned}
$$


In both above considered cases we found a subset of $\Delta_{\theta}$ of size $\Omega\left(\theta^{-2} \delta^{-f}\right)$ and dimension $O\left(\delta^{-1+f}\right)$ hence by Lemma 5 there is a regular Bohr set $B$ with $\operatorname{rk}(B) \ll \delta^{-1+f}$ and radius $\Omega\left(\delta^{1-f}\right)$ such that

$$
|(A+t) \cap B| \geqslant\left(1+\Omega\left(\theta^{2} \theta^{-2} \delta^{-f}\right)\right) \delta|B| \gg \delta^{1-f}|B|
$$

for some $t$.

Finally, if $\alpha \leqslant 20 f$ then for every $i$ we have $\left|H_{i}\right| \ll\left|\Delta_{\theta}\right|^{1 / 3-\mu / 3+\alpha+f} \leqslant\left|\Delta_{\theta}\right|^{1 / 3+21 f}, \mid H_{i}+$ $\left.H_{i}|\ll| H_{i}\right|^{1+f},\left|X_{i}\right| \ll\left|\Delta_{\theta}\right|^{2 / 3+2 \mu / 3-2 \alpha+f} \leqslant\left|\Delta_{\theta}\right|^{2 / 3+2 f}$ and

$$
\left|\left(X_{i}+H_{i}\right) \cap \Delta_{\theta}\right| \gg\left|\Delta_{\theta}\right|^{1-f},
$$

which completes the proof.

Finally, we arrived at a more difficult case, where $\Delta \approx X+H,|X| \sim \delta^{-2+O(\mu)},|H| \sim$ $\delta^{-1+O(\mu)}$ and the set $H$ is highly structured.

Lemma 13 Let $A \subseteq \mathbb{Z} / N \mathbb{Z},|A|=\delta N$, and assume that there are sets $H$ and $X$ such that $|H| \ll\left|\Delta_{\theta}\right|^{1 / 3+21 f},|H+H| \ll|H|^{1+f},|X| \ll\left|\Delta_{\theta}\right|^{2 / 3+2 f}$, and

$$
\left|(X+H) \cap \Delta_{\theta}\right| \gg\left|\Delta_{\theta}\right|^{1-21 f} .
$$

Then there is a regular Bohr set $B$ with $\operatorname{rk}(B) \leqslant \delta^{-1+f}$ and radius $\Omega\left(\delta^{1-f}\right)$ such that

$$
|(A+t) \cap B| \gg \delta^{1-f}|B|
$$

for some $t$.

Pro of. Since $\operatorname{dim}(H) \ll \delta^{-2 f}$ then there is a set $\Lambda$ such that $|\Lambda| \ll \delta^{-2 f}$ and $H \subseteq \operatorname{Span}(\Lambda)$. Let $B=B(\Lambda, \gamma)$ be a regular Bohr set with radius $1 /(6|\Lambda|) \leqslant \gamma \leqslant 1 /(3|\Lambda|)$ (the existence of such $\gamma$ is guaranteed by Lemma 4$)$. Then clearly $B \subseteq B(H, 1 / 3)$. Furthermore, for $h \in H$ and $b \in B$ we have

$$
\|h b / N\| \leqslant \sum_{\lambda \in \Lambda}\|\lambda b / N\| \leqslant 1 / 3
$$

So

$$
\left|\widehat{1_{B}}(h)\right| \geqslant \sum_{b \in B} \Re e^{-2 \pi i h b / N} \geqslant \frac{1}{2}|B| .
$$

Put $A_{t}=(A+t) \cap B$ and let us assume that for each $t$ we have

$$
\left|A_{t}\right| \ll \delta^{1-f}|B|
$$

as otherwise we would obtain the required density increment on a Bohr set with the rank $O\left(\delta^{-2 f}\right)$ and radius $\Omega\left(\delta^{2 f}\right)$. For every $x \in \mathbb{Z} / N \mathbb{Z}$ we have

$$
\widehat{1_{A_{t}}}(x)=\frac{1}{N} \sum_{h} \widehat{1_{B}}(h) \widehat{1_{A}}(x-h) e^{2 \pi i t(x-h) / N}
$$


hence by the Parseval formula

$$
\sum_{t}\left|\widehat{1_{A_{t}}}(x)\right|^{2}=\frac{1}{N} \sum_{h}\left|\widehat{1_{B}}(h) \widehat{1_{A}}(x+h)\right|^{2} \geqslant \frac{1}{N} \sum_{h \in H}\left|\widehat{1_{B}}(h) \widehat{1_{A}}(x+h)\right|^{2} .
$$

Let $Y \subseteq X$ be a set given by Lemma 11 when applied to $X, H$ and $\varepsilon=5 f$. Bounding similarly as in (11) we have

$$
|Y| \gg|X||H|^{-5 f} \gg\left|\Delta_{\theta}\right|^{2 / 3-O(f)} .
$$

Therefore, summing (13) over $Y$ we get

$$
\begin{aligned}
\sum_{t} \sum_{x \in Y}\left|\widehat{1_{A_{t}}}(x)\right|^{2} & \geqslant \frac{1}{N} \sum_{h \in H} \sum_{x \in Y}\left|\widehat{1_{B}}(h) \widehat{1_{A}}(x+h)\right|^{2} \gg \frac{1}{N}\left|(Y+H) \cap \Delta_{\theta}\right||B|^{2} \delta^{2-2 \mu}|A|^{2} \\
& \gg \delta^{3}\left|\Delta_{\theta}\right|^{1-O(f)}|B|^{2}|A| \gg \delta^{O(f)}|B|^{2}|A|
\end{aligned}
$$

Using averaging argument and (12) we see that there is a $t$ such that

$$
\sum_{x \in Y}\left|\widehat{1_{A_{t}}}(x)\right|^{2} \gg \delta^{1+O(f)}|B|^{2} \gg \delta^{-1+O(f)}\left|A_{t}\right|^{2}
$$

We can ignore all small terms in (14) that satisfy

$$
\left|\widehat{1_{A_{t}}}(x)\right| \leqslant c \frac{\delta^{-1 / 2+O(f)}}{|X|^{1 / 2}}\left|A_{t}\right|=\delta^{1 / 2+O(f)}\left|A_{t}\right|
$$

where $c>0$ is a sufficiently small constant, so by dyadic argument there is $\eta \gg \delta^{1 / 2+O(f)}$ such that

$$
\sum_{x \in Y: \eta\left|A_{t}\right| \leqslant\left|\widehat{1_{A_{t}}}(x)\right| \leqslant 2 \eta\left|A_{t}\right|}\left|\widehat{1_{A_{t}}}(x)\right|^{2} \gg \delta^{-1+O(f)} \log ^{-1}(1 / \delta)\left|A_{t}\right|^{2} \gg \delta^{-1+O(f)}\left|A_{t}\right|^{2} .
$$

Put

$$
S=\left\{x \in Y: \eta\left|A_{t}\right| \leqslant\left|\widehat{1_{A_{t}}}(x)\right| \leqslant 2 \eta\left|A_{t}\right|\right\}
$$

then by the above inequality it follows that

$$
|S| \gg \eta^{-2} \delta^{-1+O(f)} .
$$

By Lemma 6 there is a set $Z \subseteq S \subseteq Y$ such that $|Z| \geqslant \eta|S| \gg \eta^{-1} \delta^{-1+O(f)}$ and $\operatorname{dim}(Z) \ll$ $\eta^{-1} \log \left(N /\left|A_{t}\right|\right)$. From (14) one can deduce that $\left|A_{t}\right| \gg \delta^{2}|B|$ hence by Lemma 3 it follows that

$$
\left|A_{t}\right| \gg \delta^{2}|B| \geqslant \delta^{2} \gamma^{\delta^{-2 f}} N \geqslant(\delta / 8)^{2 \delta^{-2 f}} N
$$

so

$$
\operatorname{dim}(Z) \ll \eta^{-1} \delta^{-3 f} \ll \delta^{-1 / 2-O(f)} .
$$

Put $\eta_{1}=\eta$ and let $Z_{1} \subseteq Y$ be any set of size $\Theta\left(\eta_{1}^{-1} \delta^{-1+O(f)}\right)$ such that $\operatorname{dim}(Z) \ll$ $\eta_{1}^{-1} \delta^{-3 f} \ll \delta^{-1 / 2-O(f)}$. Then we apply the above argument to the set $Y \backslash Z_{1}$ to find $Z_{2} \subseteq Y \backslash Z_{1}$ and $\eta_{2} \gg \delta^{1 / 2+O(f)}$ with the same properties (observe that the whole argument can be applied 
for any set $Y^{\prime} \subseteq Y$ giving essentially the same conclusion, as long as $\left.\left|Y^{\prime}\right| \gg|Y|\right)$. Applying this procedure $k$ times we obtain disjoint sets $Z_{1}, \ldots, Z_{k} \subseteq Y$ such that $\left|Z_{i}\right|=\Theta\left(\eta_{i}^{-1} \delta^{-1+O(f)}\right)$ and $\operatorname{dim}\left(Z_{i}\right) \ll \eta_{i}^{-1} \delta^{-2 f} \ll \delta^{1-O(f)}\left|Z_{i}\right|$ for some $\eta_{i} \gg \delta^{1 / 2+O(f)}$, where $k$ is the smallest integer such that

$$
\left|Z_{1}\right|+\cdots+\left|Z_{k}\right| \geqslant \delta^{-3 / 2}
$$

Since for each $i$ we have $\left|Z_{i}\right| \leqslant \delta^{-3 / 2}$ it follows that

$$
\left|Z_{1}\right|+\cdots+\left|Z_{k}\right| \leqslant 2 \delta^{-3 / 2}
$$

Put $U=\bigcup_{i=1}^{k} Z_{i} \subseteq X$ then $|U| \geqslant \delta^{-3 / 2}$ and

$$
\operatorname{dim}(U+H) \leqslant \operatorname{dim}(H) \sum_{i=1}^{k} \operatorname{dim}\left(Z_{i}\right) \ll \delta^{1-O(f)} \sum_{i=1}^{k}\left|Z_{i}\right| \ll \delta^{-1 / 2-O(f)} .
$$

Again, by Lemma 11 we have

$$
|U+H| \gg|U||H|^{1-10 f} \gg \delta^{-5 / 2+O(f)} .
$$

Lemma[5implies that there exists a Bohr set $B^{\prime}$ with $\operatorname{rk}\left(B^{\prime}\right) \ll \delta^{-1 / 2-O(f)}$ and radius $\Omega\left(\delta^{1 / 2+O(f)}\right)$ such that

$$
\left|(A+t) \cap B^{\prime}\right| \gg\left(1+\Omega\left(\delta^{2+2 \mu} \delta^{-5 / 2+O(f)}\right)\right) \delta\left|B^{\prime}\right| \gg \delta^{1 / 2+O(f)}\left|B^{\prime}\right| \gg \delta^{1-f}\left|B^{\prime}\right|
$$

for some $t$ which is a contradiction.

\section{$7 \quad$ Large Fourier coefficients}

In this section we obtain the density increment if (21) and (3) do not hold, so there is a kind of spectral gap in terms of $L^{3}$-norm. We will use the well-known Chang's Spectral Lemma [8], which states that for every $\theta$ we have

$$
\operatorname{dim}\left(\Delta_{\theta}\right) \ll \theta^{-2} \log (1 / \delta) .
$$

For any function $f: \mathbb{Z}_{N} \rightarrow \mathbb{R}$ define

$$
T(f)=\sum_{x+y=2 z} f(x) f(y) f(2 z) .
$$

We also make use of the following lower bound on the number of 3-term arithmetic progressions in a set $S \subseteq \mathbb{Z} / N \mathbb{Z}$ with density $\gamma$ proven by Bloom [4]

$$
T\left(1_{A}\right) \geqslant \gamma^{O\left(\gamma^{-1} \log ^{4}(1 / \gamma)\right)} N^{2}
$$

Lemma 14 Let $A \subseteq \mathbb{Z} / N \mathbb{Z}$, be a set with density $\delta$ such that (2) and (3) do not hold. Then there is a regular Bohr set $B$ with $\operatorname{rk}(B) \leqslant \delta^{-2 / 5}$ and radius $\Omega\left(\delta^{4}\right)$ such that

$$
|(A+t) \cap B| \gg \mu \frac{\log (1 / \delta)}{\log \log ^{5}(1 / \delta)} \delta|B|
$$

for some $t$. 
Proof. By Chang's lemma

$$
\operatorname{dim}\left(\Delta_{\delta^{1 / 10}}\right) \ll \delta^{-1 / 5} \log (1 / \delta) \leqslant \delta^{-2 / 5}
$$

hence there is a set $\Lambda$ such that $|\Lambda| \ll \delta^{-2 / 5}$ and $\Delta_{\delta^{1 / 10}} \subseteq \operatorname{Span}(\Lambda)$. Let $B=B(\Lambda, \gamma)$ be a regular Bohr set with radius $\gamma \gg \delta^{3}$. Let $\beta=\frac{1}{|B|} 1_{B}$ then for every $r \in \Delta_{\delta^{1 / 10}}$ we have

$$
|\widehat{\beta}(r)-1| \leqslant \frac{1}{|B|} \sum_{b \in B}\left|e^{-2 \pi i \lambda b / N}-1\right| \leqslant \frac{2 \pi}{|B|} \sum_{b \in B} \sum_{\lambda \in \Lambda}\|r b / N\| \leqslant 2 \pi \delta^{2}
$$

and similarly $|\widehat{\beta}(2 r)-1| \ll \delta^{2}$. Let $f: \mathbb{Z}_{N} \rightarrow[0,1]$ be a function defined by

$$
f(t)=\beta * 1_{A}(t) .
$$

We may assume that $f(t)=\frac{1}{|B|}|(A+t) \cap B| \leqslant L \delta$, where

$$
L=c \mu \frac{\log (1 / \delta)}{\log \log ^{5}(1 / \delta)}
$$

and $c>0$ is a small constant. Put

$$
S=\{t: f(t) \geqslant \delta / 2\}
$$

then by $\sum_{t} f(t)=|A|$ it follows that $|S| \geqslant N / L$ hence by Bloom's Theorem we have

$$
T(f) \geqslant \frac{1}{8} \delta^{3} T(S) \gg \delta^{3} \exp \left(-O\left(L \log ^{5} L\right)\right) N^{2} \gg \delta^{3+\mu / 10} N^{2} .
$$

Our next step is to compare $T(f)$ and $T\left(1_{A}\right)$. By (16), (2), (3), Parseval's formula and Hölder's inequality we have

$$
\begin{aligned}
\left|T\left(1_{A}\right)-T(f)\right| & =\frac{1}{N}\left|\sum_{r=0}^{N-1} \widehat{1_{A}}(r)^{2} \widehat{1_{A}}(-2 r)-\sum_{r=0}^{N-1} \widehat{f}(r)^{2} \widehat{f}(-2 r)\right| \\
& \leqslant \frac{1}{N} \sum_{r \in \Delta_{\delta^{1 / 10}}}\left|\widehat{1_{A}}(r)^{2} \widehat{1_{A}}(-2 r)\left(1-\widehat{\beta}(r)^{2} \widehat{\beta}(-2 r)\right)\right|+\frac{2}{N} \sum_{r \notin \Delta_{\delta^{1 / 10}}}\left|\widehat{1_{A}}(r)\right|^{3} \\
& \ll \delta^{2} \frac{1}{N} \sum_{r \in \Delta_{\delta^{1 / 10}}}\left|\widehat{1_{A}}(r)\right|^{3}+\frac{2}{N} \sum_{r \in \Delta_{\delta^{1+\mu} \backslash \Delta_{\delta^{1 / 10}}}}\left|\widehat{1_{A}}(r)\right|^{3}+\frac{2}{N} \sum_{r \notin \Delta_{\delta^{1+\mu}}}\left|\widehat{1_{A}}(r)\right|^{3} \\
& \ll \delta^{2}|A|^{2}+\delta^{1+\mu / 5}|A|^{2}+2 \delta^{1+\mu}|A|^{2} \ll \delta^{3+\mu / 5} N^{2} .
\end{aligned}
$$

Thus, by (17)

$$
T\left(1_{A}\right) \gg \delta^{3+\mu / 10} N^{2},
$$

which is a contradiction. 


\section{Proof of Theorem 1}

Summarizing all considered cases, we can state the following result.

Theorem 15 There exists an absolute constant $c>0$ such that the following holds. Let $A \subseteq$ $\mathbb{Z} / N \mathbb{Z}$ be a set without any non-trivial arithmetic progressions of length three and let $|A|=\delta N$. Then there is a regular Bohr set $B$ with $\operatorname{rk}(B) \ll \delta^{-1+c}$ and radius $\Omega\left(\delta^{4}\right)$ such that for some $t$

$$
|(A+t) \cap B| \gg \frac{\log (1 / \delta)}{\log \log ^{5}(1 / \delta)} \delta|B|
$$

Proof. Let us first make a suitable choice of parameters. Let $\mu>0$ be a constant the such that for every $\sigma \leqslant \mu$, (15) holds with $f=f(16 \sigma)$. Since we assumed that $f(\mu) \geqslant \mu$, we see that in all considered cases in Lemma 7, Lemma 9, Lemma 12, Lemma 13 and Lemma 14 we obtain density increment at least by factor of $\Omega\left(\mu \log (1 / \delta) \log \log ^{-5}(1 / \delta)\right)$ on a Bohr set with $\operatorname{rk}(B) \ll \delta^{-1+\mu / 3}$ and radius $\Omega\left(\delta^{4}\right)$. Thus, it is enough to take $c=\mu / 3$.

After the first step of our iterative procedure we obtain a larger density increment on a low-rank Bohr set and then we apply less effective method of Bloom (Theorem 7.1 [4]).

Lemma 16 [4] There exists an absolute constant $c_{1}>0$ such that the following holds. Let $B \subseteq \mathbb{Z} / N \mathbb{Z}$ be a regular Bohr set of rank d. Let $A_{1} \subseteq B$ and $A_{2} \subseteq B_{\varepsilon}$, each with relative densities $\alpha_{i}$. Let $\alpha=\min \left(c_{1}, \alpha_{1}, \alpha_{2}\right)$ and assume that $d \leqslant \exp \left(c_{1}\left(\log ^{2}(1 / \alpha)\right)\right.$. Suppose that $B_{\varepsilon}$ is also regular and $c_{1} \alpha /(4 d) \leqslant \varepsilon \leqslant c_{1} \alpha / d$. Then either

(i) there is a regular Bohr set $B^{\prime}$ of rank $\operatorname{rk}\left(B^{\prime}\right) \leqslant d+O\left(\alpha^{-1} \log (1 / \alpha)\right)$ and size

$$
\left|B^{\prime}\right| \geqslant \exp \left(-O\left(\log ^{2}(1 / \alpha)\left(d+\alpha^{-1} \log (1 / \alpha)\right)\right)\right)|B|
$$

such that

$$
\left|\left(A_{1}+t\right) \cap B^{\prime}\right| \gg\left(1+c_{1}\right) \alpha_{1}\left|B^{\prime}\right|
$$

for some $t \in \mathbb{Z} / N \mathbb{Z}$;

(ii) or there are $\Omega\left(\alpha_{1}^{2} \alpha_{2}|B|\left|B_{\varepsilon}\right|\right)$ three-term arithmetic progressions $x+y=2 z$ with $x, y \in$ $A_{1}, z \in A_{2}$;

Now we are in position to finish the proof of our main result. We will not give detailed proof of the iteration procedure as it is very standard and the reader can find details on it in the literature (see [4], 21]). In the first step we apply Theorem 15 to obtain a regular Bohr set $B^{0}$ with $\operatorname{rk}\left(B^{0}\right) \ll \delta^{-1+c}$, radius $\Omega\left(\delta^{4}\right)$ and a progression-free set $A_{0} \subseteq A+t$ for some $t$ such that

$$
\left|A_{0} \cap B^{0}\right| \gg \alpha\left|B^{0}\right|
$$

where

$$
\alpha \gg \frac{\log (1 / \delta)}{\log \log ^{5}(1 / \delta)} \delta
$$


By Lemma 3 we have

$$
\left|B^{0}\right| \geqslant \exp \left(-O\left(\delta^{-1+c} \log (1 / \delta)\right)\right) N .
$$

Next we iteratively apply Lemma[16 and let $B^{i}$ be Bohr sets obtained in the iterative procedure. Observe that after $k \ll \log (1 / \alpha)$ steps we will be in the case $(i i)$ of Lemma 16 and that $\operatorname{rk}\left(B^{i}\right) \ll \alpha^{-1} \log ^{2}(1 / \alpha)$ for every $i \leqslant k$. Thus, there are

$$
\Omega\left(\alpha^{3}\left|B^{k}\right|\left|B_{\varepsilon}^{k}\right|\right)
$$

three-term arithmetic progressions in $A$, where $\varepsilon \geqslant c_{1} \alpha /\left(4 \operatorname{rk}\left(B^{k}\right)\right) \gg \alpha^{2} \log ^{2}(1 / \alpha)$.

Hence by Lemma 16 we have

$$
\left|B^{k}\right| \geqslant \exp \left(-O\left(\alpha^{-1} \log ^{4}(1 / \alpha)\right)\right) N \geqslant \exp \left(-O\left(\delta^{-1} \log ^{3}(1 / \delta)\right) \log \log ^{5}(1 / \delta)\right) N,
$$

and by Lemma 3

$$
\begin{aligned}
\left|B_{\varepsilon}^{k}\right| & \geqslant \exp \left(-O\left(\alpha^{-1} \log ^{3}(1 / \alpha)\right)\right) \exp \left(-O\left(\alpha^{-1} \log ^{4}(1 / \alpha)\right)\right) N \\
& \geqslant \exp \left(-O\left(\delta^{-1} \log ^{3}(1 / \delta) \log \log ^{5}(1 / \delta)\right)\right) N
\end{aligned}
$$

Therefore $A$ contains

$$
\alpha^{3} \exp \left(-O\left(\delta^{-1} \log ^{3}(1 / \delta) \log \log ^{5}(1 / \delta)\right)\right) N^{2}
$$

arithmetic progressions of length three. Since there are only $|A|$ trivial progressions it follows that

$$
|A| \geqslant \alpha^{3} \exp \left(-O\left(\delta^{-1} \log ^{3}(1 / \delta) \log \log ^{5}(1 / \delta)\right)\right) N^{2},
$$

which completes the proof of Theorem 1 .

\section{Concluding remarks}

In Lemma 7, Lemma 9, Lemma 12 and Lemma 13 we obtained a density increment by factor of $\delta^{-c}$ on a low-rank Bohr set, where $c$ is a positive constant. Such density increment even in the first step of an iterative method would lead to the upper bound $O\left((\log N)^{-1-c}\right)$ in Theorem 1 . However, in Lemma 14 we only were able to prove an increment by factor $(\log (1 / \delta))^{1-o(1)}$. Any refinement of Lemma 14 will directly imply an improvement of Theorem 1.

\section{References}

[1] M. Bateman, N. Katz, New bounds on cap sets, Journal of AMS 2 (2012), 585-613.

[2] M. Bateman, N. KATZ, Structure in additively nonsmoothing sets, arXiv:1104.2862v1 [math.CO] 14 Apr 2011.

[3] F. A. Behrend, On sets of integers which contain no three terms in arithmetical progression, Proc. Nat. Acad. Sci. U. S. A. 32, (1946). 331-332. 
[4] T. Bloom, A quantitative improvement for Roth's theorem on arithmetic progressions, J. Lond. Math. Soc. 93 (2016), 643-663.

[5] T. Bloom, O. Sisask, Logarithmic bounds for Roth's theorem via almost-periodicity, Discrete Analysis 4 (2019), 20 pp.

[6] J. Bourgain, On triples in arithmetic progression, Geom. Funct. Anal. 9 (1999), 968-984.

[7] J. Bourgain, Roth's Theorem on Progressions Revisited, J. Anal. Math. 104 (2008), 155206.

[8] M.-C. Chang, A polynomial bound in Freiman's theorem, Duke Math. J. 3 (2002), 399419 .

[9] E. Croot, V. Lev, P. PACH, Progression-free sets in $\mathbb{Z}_{4}^{n}$ are exponentially small, Ann. of Math. 185 (2017), 331-337.

[10] M. Elkin, An Improved Construction of Progression-Free Sets, Israel J. Math. 184 (2011), 93-128.

[11] J. Ellenberg, D. GijswiJt, On large subsets of $\mathbb{F}_{q}^{n}$ with no three-term arithmetic progression Ann. of Math. 185 (2017), 339-343.

[12] B. Green, Roths theorem in the primes, Ann. of Math. 161 (2005), 1609-1636.

[13] B. Green and J. Wolf, A note on Elkin's improvement of Behrend's construction, in Additive Number Theory (Springer, New York, 2010), 141-144.

[14] D. R. Heath-Brown, Integer sets containing no arithmetic progressions, J. Lond. Math. Soc. 35 (1987), 385-394.

[15] H. Helfgott And A. De Roton, Improving Roths theorem in the primes, Int. Math. Res. Not. IMRN 4 (2011), 767-783.

[16] E. Naslund, On improving Roth's theorem in the primes, Mathematika 61 (2015), 49-62.

[17] J. Pintz, W. L. Steiger, E. Szemerédi, On sets of natural numbers whose difference set contains no squares, J. London Math. Soc. 37 (1988), 219-231.

[18] K. F. Roth, On certain sets of integers, J. London Math. Soc. 28 (1953), 104-109.

[19] R. Salem, D. C. Spencer, On sets of integers which contain no three terms in arithmetical progression, Proc. Nat. Acad. Sci. Wash., 28 (1942), 561 - 563.

[20] T. Sanders, On a theorem of Shkredov, Online J. Anal. Comb. No. 5 (2010), Art. 5, 4 pp.

[21] T. Sanders, On certain other sets of integers, J. Anal. Math. 116 (2012), 53-82.

[22] T. Sanders, On Roth's Theorem on Progressions, Ann. of Math. 174 (2011), 619-636. 
[23] T. Schoen, I. D. Shkredov, Additive dimension and a theorem of Sanders, J. Aust. Math. Soc. 100 (2016), 124-144.

[24] T. Schoen, I. D. Shkredov, Roth's theorem in many variables, Israel J. Math. 199 (2014), $287-308$.

[25] T. Schoen, O. Sisask, Roth's theorem for four variables and additive structures in sums of sparse sets, Forum Math. Sigma 4 (2016), e5, 28 pp.

[26] E. SzemeréDI, Integer sets containing no arithmetic progressions, Acta Math. Hungar. 56 (1990), 155-158.

[27] E. SzEMERÉDI, Integer sets containing no $k$ elements in arithmetic progression, Acta Arith. 27 (1975), 199-245.

[28] I. D. Shkredov, On sets of large trigonometric sums, (Russian) Izv. Ross. Akad. Nauk Ser. Mat. 72 (2008), no. 1, 161-182; translation in Izv. Math. 72 (2008), 149-168.

[29] I. D. Shkredov, On sumsets of dissociated sets, Online J. Anal. Comb. No. 4 (2009), 26 pp.

[30] I. D. Shkredov, S. Yekhanin, Sets with large additive energy and symmetric sets, Journal of Combinatorial Theory, Series A 118 (2011), 1086-1093.

[31] T. Tao, V. Vu, Additive combinatorics, Cambridge University Press 2006.

Faculty of Mathematics and Computer Science,

Adam Mickiewicz University,

Umultowska 87, 61-614 Poznań, Poland

schoen@amu.edu.pl 